\title{
Age and sex interactions in gastric cancer incidence and mortality trends in Korea
}

\author{
Minkyo Song • Daehee Kang $\cdot$ Jae Jeong Yang $\cdot$ Ji-Yeob Choi $\cdot$ Hyuna Sung $\cdot$ \\ Yunhee Lee $\cdot$ Hyung-Suk Yoon $\cdot$ Yunhee Choi $\cdot$ Seong-Ho Kong $\cdot$ \\ Hyuk-Joon Lee $\cdot$ Han-Kwang Yang $\cdot$ Woo Ho Kim
}

Received: 17 February 2014/Accepted: 22 July 2014/Published online: 5 August 2014

(C) The International Gastric Cancer Association and The Japanese Gastric Cancer Association 2014

\begin{abstract}
Background The incidence and mortality of gastric cancer have declined rapidly over the past few decades, but reverse trends in the young generation's differential between sexes in some races have been reported in recent years. To investigate whether this divergent trend can be observed in Korea, a country with the highest incidence in the world, age- and sex-specific incidence and mortality trends were evaluated.

Methods Gastric cancer incidence data for the years 1999-2010 and mortality data for the years 1983-2012 were obtained from the Korea Central Cancer Registry and National Statistical Office. Annual percentage changes were calculated by age group (20-39, 40-54, 55-69, 70-79) using Joinpoint regression analysis. Furthermore, age-period-cohort analysis was evaluated.

Results Overall age-standardized gastric cancer incidence in adults aged between 20 and 79 was declining at a
\end{abstract}

M. Song · D. Kang · J. J. Yang · Y. Lee · H.-S. Yoon Department of Preventive Medicine, Seoul National University College of Medicine, Seoul, Korea

M. Song · D. Kang - J.-Y. Choi · Y. Lee · H.-S. Yoon Department of Biomedical Sciences, Seoul National University College of Medicine, Seoul, Korea

M. Song $\cdot$ J. J. Yang $\cdot$ H.-S. Yoon

Institute of Environmental Medicine, Seoul National University

Medical Research Center, Seoul, Korea

D. Kang $(\bowtie) \cdot$ J.-Y. Choi $\cdot$ S.-H. Kong $\cdot$ H.-J. Lee ·

H.-K. Yang

Department of Preventive Medicine, Cancer Research Institute,

Seoul National University College of Medicine, 103 Yongon

(Daehangno), Jongno-gu, Seoul, Korea

e-mail: dhkang@snu.ac.kr nonsignificant average annual percentage change (AAPC) of $-0.2 \%$ in males and $-0.4 \%$ in females in Korea during 1999-2010. Mortality was steeper at a significant rate of -4.3 and $-5.9 \%$ in males and females, respectively. However, age-specific analysis revealed a flat (males $0.2 \%, 95 \% \mathrm{CI}-0.5-0.6$ ) or increasing incidence trend (females $1.7 \%, 95 \%$ CI $0.9-2.5$ ) in the 40-54 age group. APC analysis confirmed the decreasing incidence and mortality trend mainly by the cohort effect.

Conclusions The differential pattern between males and females in different age groups suggests a possible effect of detection by screening practice or a signal of the change in epidemiological factors in the incidence and mortality of gastric cancer that warrants further studies.

Keywords Stomach cancer - Epidemiology - Incidence · Mortality · Trend

\author{
H. Sung \\ Division of Epidemiology and Genetics, National Cancer \\ Institute, Rockville, MD, USA \\ Y. Choi \\ Medical Research Collaborating Center, Seoul National \\ University Hospital, Seoul, Korea \\ S.-H. Kong · H.-J. Lee $\cdot$ H.-K. Yang \\ Department of Surgery, Seoul National University College of \\ Medicine, Seoul, Korea \\ W. H. Kim \\ Department of Pathology, Seoul National University College of \\ Medicine, Seoul, Korea
}




\section{Introduction}

Despite its marked decline over the past few decades, gastric cancer is still the third leading cause of cancer death and the fifth most common cancer worldwide [1]. Although the overall rate has shown a dramatic decline, which Howson et al. called the "epidemiology of an unplanned triumph," the absolute number of new cases per year is increasing, causing almost 1 million new cases in 2012, mainly because of the aging of the world population $[1,2]$.

However, the overall rate, which is an estimate by agestandardizing to the reference population, obscures the important age-specific differences. The age-standardized rate is mostly driven by the higher rates in older generations, which consequently could mask the rates in recent birth cohorts and younger generations [3]. Indeed, several studies from the Western population have recently started to report divergent trends in subgroup populations according to age group, race or anatomical subtype [4-9]. In addition to the age-specific rates, sex-specific analysis is also critical for viewing trends and associations in epidemiology, especially in gastric cancer, which is one of the most well-known cancers in which the incidence and mortality are different between sexes, usually twice as high in males compared to females [1].

Despite the importance of this issue, so far only a few studies have quantified the age- and sex-specific differences in trends in the incidence and mortality. Some were limited by the sample size for analyzing both age- and sexspecific trends [5, 7]; others were based on geographically restricted cancer registries [10]. In Asia, to our knowledge, only one study by Liu et al. has reported age-specific incidence trends, yet the study was based on a registry that covers only 10-20\% of all gastric cancers in Japan, mostly restricted to operated patients (resection rate as high as $95 \%$ ) [11]. In Korea, so far no studies have looked into the age- and sex-specific rates in gastric cancer [12-15]. Consequently, in this article, we aim to evaluate the ageand sex-specific trend of gastric cancer incidence and mortality over the last 30 years in Korea, where the incidence is the highest in the world.

\section{Materials and methods}

\section{Data sources}

Cancer cases are from the cancer registry in Korea, which began in 1975 . The registry had been collecting information on an estimated $80-90 \%$ of cancer cases from more than 150 training hospitals across the country until 1999, when it expanded the cancer registry to cover the entire Korean population under the Population-Based Regional
Cancer Registry (PBCR) program [16]. For analysis, gastric cancer cases were defined and extracted using the International Classification of Diseases, 10th edition (ICD10), code C16. The 5-year-interval age- and sex-specific number of incident gastric cases between 1999 and 2010 and the number of deaths for gastric cancer between 1983 and 2012, as well as the population numbers, which are the estimated yearly values from the census data, were obtained from the Korea Statistical Information System (KOSIS) of the National Statistical Office in Korea [17].

\section{Statistical methods}

Annual age-specific incidence and mortality rates $(100,000$ person-years) were calculated for the ages 20-79. Temporal trends were assessed by the annual percent change (APC) by least square linear regression using the Joinpoint Regression Program version 4.0.4 (Statistical Research and Applications Branch, National Cancer Institute, NCI, Rockville, MD, USA). Average annual percentage changes (AAPCs) were calculated for the entire study period. To facilitate the statistical precision for tabular presentation, the 125 -year age groups were further combined into the 20-39, 40-54, 55-69 and 70-79 year age groups from the Appendix. Furthermore, in Korea, age 40 is the starting point in the national screening program, and age 55 is the average age for retirement for most of the companies. In the analysis of the collapsed age groups, direct age standardization using the Segi population was performed for each age group following the standardization method for truncated age-standardized rates (ASR) [18]. To calculate the standard error (SE) of the direct ASR for the APC analysis, Poisson approximation was used with the following formula [19].

$\operatorname{Var}(\mathrm{ASR})=\frac{\sum_{i=1}^{A}\left[\frac{a_{i} w_{i}^{2} \times 100,000}{n_{i}}\right]}{\left(\sum_{i=1}^{A} w_{i}\right)^{2}}$

In the formula, where $i=1,2, \ldots \mathrm{A}, a$ is age class $a_{i}$ representing the age-specific rate in the $i$ th age class, $n_{i}$ is person-years of observation for the corresponding age class, and $w_{i}$ is the $i$ th age class of the standard population. Furthermore, generalized linear regression models were used with $\log$ person years set as an offset, assuming Poisson distribution, to test whether time trends varied by age group (20-39, 40-54, 55-69 and 70-79 years) or sex. To test for age group and year interaction the four age groups and number of years (11 for incidence and 30 for mortality) were entered in the interaction model. Similarly, to seek for age group and sex interaction, four age groups, two sexes and their interaction term were put into the model. 
To further characterize the variations of incidence and mortality rates by age, period and birth cohort effects by sex, age-period-cohort (APC) analysis was evaluated. For the incidence data, we interpolated single-year data for both cases and the population from 5-year interval periods, assuming a linear trend for each interval, and further grouped the single-year data into 31 2-year age groups $(19-20,20-21, \ldots, 79-80)$ and 9 2-year time periods $(1999-2000,2001-2002, \ldots, 2009-2010)$. To minimize the assumption of a linear relationship in interpolating the 2-year age groups, linear assumption was applied to two adjacent known data points in a piecewise manner, not to the entire distribution. To sufficiently capture the real distribution, linear interpolation was carried out separately for cumulative personyears and cumulative numbers of events with cubic spine function for smoothing the place where polynomial pieces connect. For mortality, 6 equally spaced 5-year calendar periods (1983-1987, 1988-1992, ..., 2008-2012) and 12 5-year age groups $(20-24,25-29, \ldots, 75-79)$ were used for the analysis. The models were sequentially presented as a one-factor age model, two-factor age drift, age cohort, age-period model and finally the full ageperiod-cohort model. The deviances of the models were compared to evaluate the statistical significance, and the goodness of fit, presented as adjusted $R^{2}$, was computed to measure the variability explained by the factors other than age. All analyses were performed separately for males and females.

All other statistical analyses besides APC analysis were performed in SAS software version 9.3 (SAS Institute, Inc. Cary, NC). APC was executed with R software version 3.0.1 using the 'Epi' package, version 1.1.49, and model fitting with the apc.fit function [20]. The model was estimated using parametric smooth functions based on natural splines with five knots each for age, period and cohort variables to encounter nonlinear effects, with the median date of birth as reference. Maximum-likelihood fit (ML-fit) of the APC model had rendered the goodness of fit scaled by deviance. The graphic display of the APC model was performed with function apc.plot and apc.lines, with age effects as rates for the reference cohort using "ACP" as the parm value in parametrization of the effect in the apc.fit function to emphasize the cohort effect of the fitted APC model. In this model, the cohort effects are represented as the rate ratio (RR) relative to the reference cohort, and period effects are constrained to be zero on average with zero slope. All analyses were performed at a two-sided significance level of $p<0.05$. The term APC denotes both annual percent change and age period cohort; thus, to avoid any confusion in the article, APC is confined to age period cohort and annual percent change is only used as AAPC as its acronym.

\section{Results}

In $417,896,466$ person-years of observation, a total of 280,755 incident cases were diagnosed during 1999-2010. The overall age-standardized incidence rate per 100,000 person-years decreased from 107.54 in 1999 to 103.07 in 2010 in males and 44.30 in 1999 to 41.43 in 2010 in females (data not shown). Among the ages $20-79$, the agestandardized incident rate was 99.89 in males and 40.06 in females per 100,000 person-years (Table 1). The agestandardized incidence rate was higher in females in the ages $20-39$ (7.40 in 100,000 males compared to 8.33 in 100,000 females), but in those aged 40 years and older, males had a higher rate than females. It was about twice as high in males as in females, ranging from 2.08 in the 40-54 age group, peaking at 55-69 to 3.02 and narrowing to 2.66 in the 70-79 age group. The overall trend for ages 20-79 expressed in AAPC decreased at a rate of -0.2 and $-0.4 \%$ in males and females, respectively, although this was not statistically significant during the 12-year study period. In the age-specific analysis, the most significant decrease was observed for the 20-39 age group at a rate of $-3.7 \%$ for males, whereas in females there was a nonsignificant decrease of $-0.8 \%$. For the $40-54$ age group, in both sexes there was a positive change in trend expressed as AAPC of $0.2 \%$ [95\% confidence interval (CI) $-0.2-0.6]$ in males and $1.7 \%$ (95\% CI 0.9-2.5) in females, in contrast to other age groups that showed negative decreasing trends. Consequently, despite the overall decrease in trends, the incidence trend varied among age groups in both males and females $(p<0.0001$ for age group $\times$ year interaction).

For mortality rates, in the $930,932,796$ person-years observed, a total of 300,901 deaths were identified during 1983-2012. The overall age-standardized rate decreased from 105.73 in 1983 to 28.23 in 2012 in males and 47.97 to 10.64 in females during the same period (data not shown). Among those aged 20-79, the age-standardized mortality rate was 52.75 in males and 21.63 in females per 100,000 person-years (Table 1). The age-standardized mortality rate was higher in females aged 20-39 (3.85 in 100,000 males compared to 4.58 in 100,000 females), but in those aged 40 years and older, males had a higher rate than females. The rate was twice as high in males as in females, ranging from 1.96 in the $40-54$ age group, peaking at 55-69 to 2.92 and narrowing to 2.69 in the 70-79 age group, a similar pattern to that observed in incidence rates. The overall mortality was decreasing at a faster rate than the incidence rate of AAPC of -4.3 and $-5.9 \%$ for males and females, respectively. Age group-specific analysis showed decreasing trends in all age groups, with statistically significant ranges from -2.7 to $-6.9 \%$. Despite the overall decrease in trends, the mortality trend varied among 
Table 1 Number of cases, ageadjusted incidence and mortality rates per 100,000 person-years and AAPCs of gastric cancer for selected age groups by sex in Korea during 1999-2010 (incidence) and 1983-2012 (mortality)

\begin{tabular}{|c|c|c|c|}
\hline \multicolumn{2}{|l|}{ No. of cases } & \multirow[t]{2}{*}{ Rate per 100,000 person-years $(95 \% \mathrm{CI})$} & \multirow[t]{2}{*}{ AAPC \% $(95 \%$ CI $)$} \\
\hline \multicolumn{2}{|l|}{ Incidence } & & \\
\hline \multicolumn{4}{|l|}{ Males } \\
\hline $20-39$ & 8,573 & $7.40(7.24-7.56)$ & $-3.7 *(-4.5$ to -2.8$)$ \\
\hline $40-54$ & 46,682 & $73.11(72.45-73.77)$ & $0.2 *(-0.2$ to 0.6$)$ \\
\hline $55-69$ & 91,616 & $278.60(276.79-280.41)$ & $-0.1 *(-0.6$ to 0.5$)$ \\
\hline $70-79$ & 42,982 & $459.59(459.50-467.37)$ & $-0.5^{*}(-1.0$ to 0.1$)$ \\
\hline Overall & 189,853 & 99.89 (99.44-100.34) & $-0.2 *(-0.6$ to 0.2$)$ \\
\hline \multicolumn{4}{|l|}{ Females } \\
\hline $20-39$ & 8,986 & $8.33(8.16-8.51)$ & $-0.8^{*}(-1.7$ to 0.2$)$ \\
\hline $40-54$ & 22,166 & $35.13(34.66-35.59)$ & $1.7 *(0.9$ to 2.5$)$ \\
\hline $55-69$ & 34,267 & $92.20(91.22-93.18)$ & $-1.1 *(-1.6$ to -0.6$)$ \\
\hline $70-79$ & 25,483 & $172.86(170.71-175.01)$ & $-1.0 *(-1.6$ to -0.4$)$ \\
\hline Overall & 90,902 & $40.06(39.79-40.32)$ & $-0.4 *(-0.9-0.1)$ \\
\hline \multicolumn{4}{|l|}{ Mortality } \\
\hline \multicolumn{4}{|l|}{ Males } \\
\hline 20-39 & 10,046 & $3.85(3.77-3.92)$ & $-6.9 *(-8.3$ to -5.4$)$ \\
\hline $40-54$ & 43,182 & $33.00(32.69-33.31)$ & $-6.1 *(-6.5$ to -5.7$)$ \\
\hline $55-69$ & 92,119 & $141.04(140.12-141.96)$ & $-5.3 *(-5.8$ to -4.8$)$ \\
\hline $70-79$ & 53,318 & $297.26(294.73-299.79)$ & $-2.7 *(-3.6$ to -1.8$)$ \\
\hline Overall & 198,665 & $52.75(52.51-52.98)$ & $-4.3^{*}(-5.2$ to -4.4$)$ \\
\hline \multicolumn{4}{|l|}{ Females } \\
\hline $20-39$ & 11,223 & $4.58(4.49-4.66)$ & $-5.5^{*}(-6.3$ to -4.7$)$ \\
\hline $40-54$ & 21,728 & $16.80(16.58-17.02)$ & $-5.6^{*}(-5.9$ to -5.3$)$ \\
\hline $55-69$ & 37,251 & $48.26(47.77-48.75)$ & $-6.5^{*}(-6.9$ to -6.1$)$ \\
\hline $70-79$ & 32,034 & $110.61(109.39-111.84)$ & $-3.8 *(-5.1$ to -2.5$)$ \\
\hline Overall & 102,236 & $21.63(21.50-21.77)$ & $-5.9 *(-6.2$ to -4.9$)$ \\
\hline
\end{tabular}

age groups in both males and females $(p<0.0001$ for age group $\times$ year interaction).

Figure 1 shows the yearly incidence and mortality rate for each age group. Overall, the incidence rate gradually decreased, whereas the mortality rate showed a steep decrease in most of the age groups. Due to the difference in magnitude of change in AAPC, the yearly change differs between males and females of the same age groups, shown as $p$-interaction values for sex $\times$ year in the figure.

The summary statistics for the APC model are presented in the Appendix. All of the drift, period and cohort effects were statistically significantfor incidence after adjusting for age $(p<0.0001)$ except for the APC model (PIAC) in females. The adjusted $R^{2}$ values were greatly increased for the age-cohort model compared to the insignificant increase in the age-period model for both sexes. However, although the trend in incidence is dominantly explained by the age cohort model even after adjusting for the period effects, the full model had to fit better to be chosen as the final model. For mortality, the age-drift model alone could explain about 86 and $84 \%$ of gastric cancer mortality in males and females, respectively. However, after adjustment for age, both the period and cohort effect showed larger adjusted $R^{2}$ values in both sexes. Particularly the addition of the cohort term had a more incremental effect on the adjusted $R^{2}$ values, from 0.86 to 0.98 in males and from 0.84 to 0.98 in females. However, similar to the incidence model, the full APC model further increased the adjusted $R^{2}$ value by 0.01 and was chosen as the final model.

A graphic display of the APC model is presented in Fig. 2 for the reference cohorts. For incidence, the reference cohort was set to 1942 for both sexes; the age-specific rate showed an increase in the incidence rate in older age groups, males at a steeper rate than females. The cohort relative risks in the middle showed the incidence in males was steadily decreasing. When compared to the 1942 cohort, the 1972 birth cohorts had a $30 \%$ decreased risk of gastric cancer incidence in males $(\mathrm{RR}=0.70,95 \% \mathrm{CI}$ 0.66-0.75). In females, the cohorts born between 1960 and 1980 were at increased risk of gastric cancer incidence compared to the 1942 cohorts. The females born after 1980 were at decreased risk of gastric cancer incidence. For mortality, risk increased with age in males at a faster rate than in females; a similar pattern was observed for the 
Fig. 1 Age standardized rate in $\log$ scale of gastric cancer incidence (a) and mortality (b) in males and females in different age groups during 1999-2010 (incidence) and 1983-2012 (mortality) in Korea
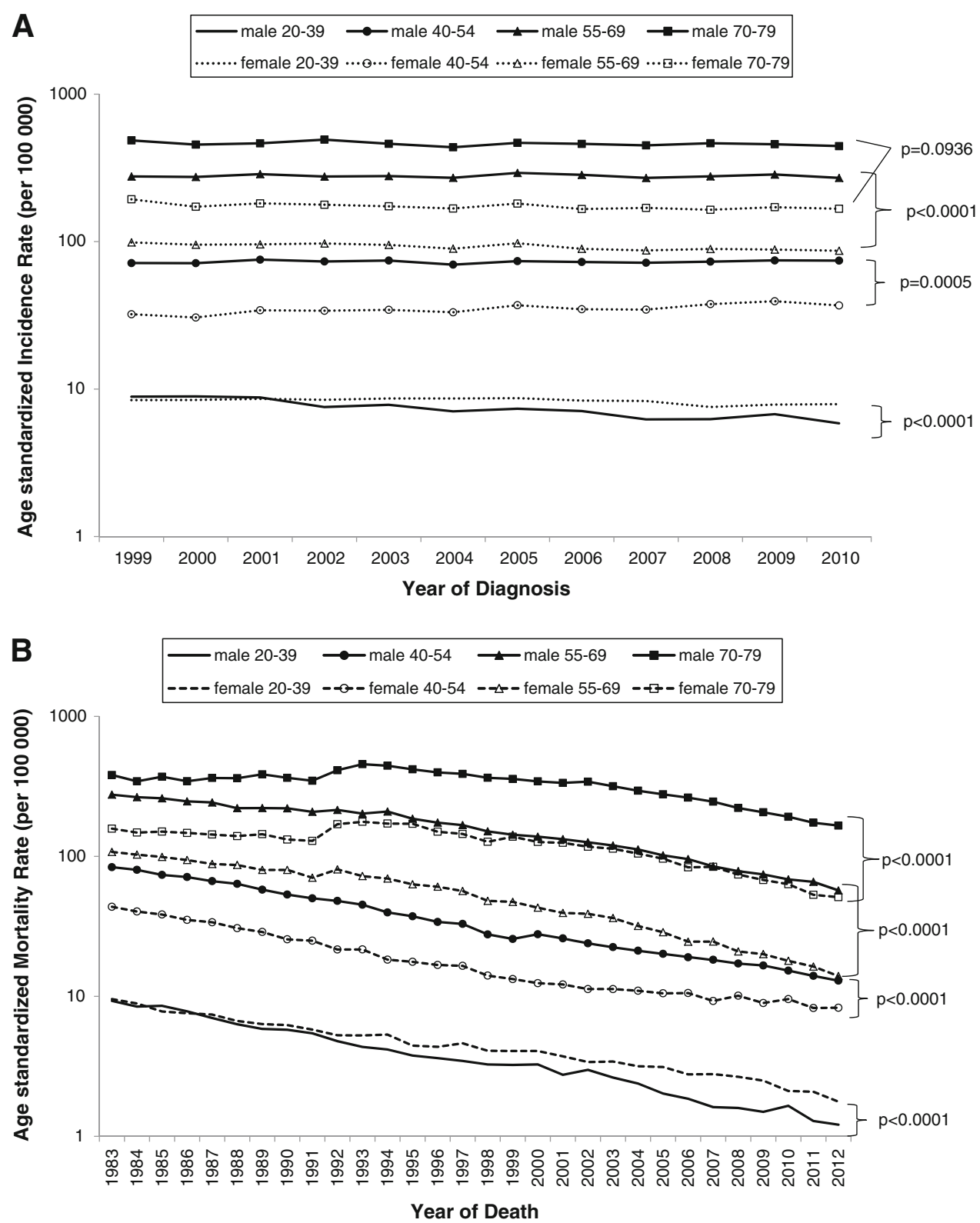

incidence rate. The rate ratios for all the cohorts were decreasing compared to the reference cohort born in 1933 for both males and females. Interestingly, females born in 1950-1970 did not experience a rapid decline; it was observed as a more gradual change in the rate ratio.

\section{Discussion}

Our analysis focused on the age- and sex- specific incidence and mortality rates for gastric cancer in the Korean population using the nationally representative registry and mortality data. The overall trend decreased for both incidence and mortality in both sexes, but the generations between the ages 40 and 54 experienced an increasing incidence trend, more significantly in females. Furthermore, differences in the rate of change between the sexes (i.e., sex $\times$ year interaction) were observed in most of the age groups, most pronounced in the youngest age group of 20-39. Moreover, through the APC analysis with age effect adjusted, the present study confirms the decreasing trends in recent cohorts, males born after 1960 and females born after 1980. In accordance with the AAPC results, mortality rates have been in a steep decline in both males and females, although the rates differ between the sexes.

Previously, a reversal of the downward trend has been observed in some of the youngest generations. As in the case of incidence, an earlier study from Spain reported 
Fig. 2 Age-period-cohort effects on trends in gastric cancer incidence and mortality: Korea, 1999-2010 for incidence (a) and 1983-2012 for mortality (b) (open circles refer to the reference group for period. Adjusted risk ratio for the reference cohort 1942 for incidence and 1933 for mortality; the median date of birth for persons with events)
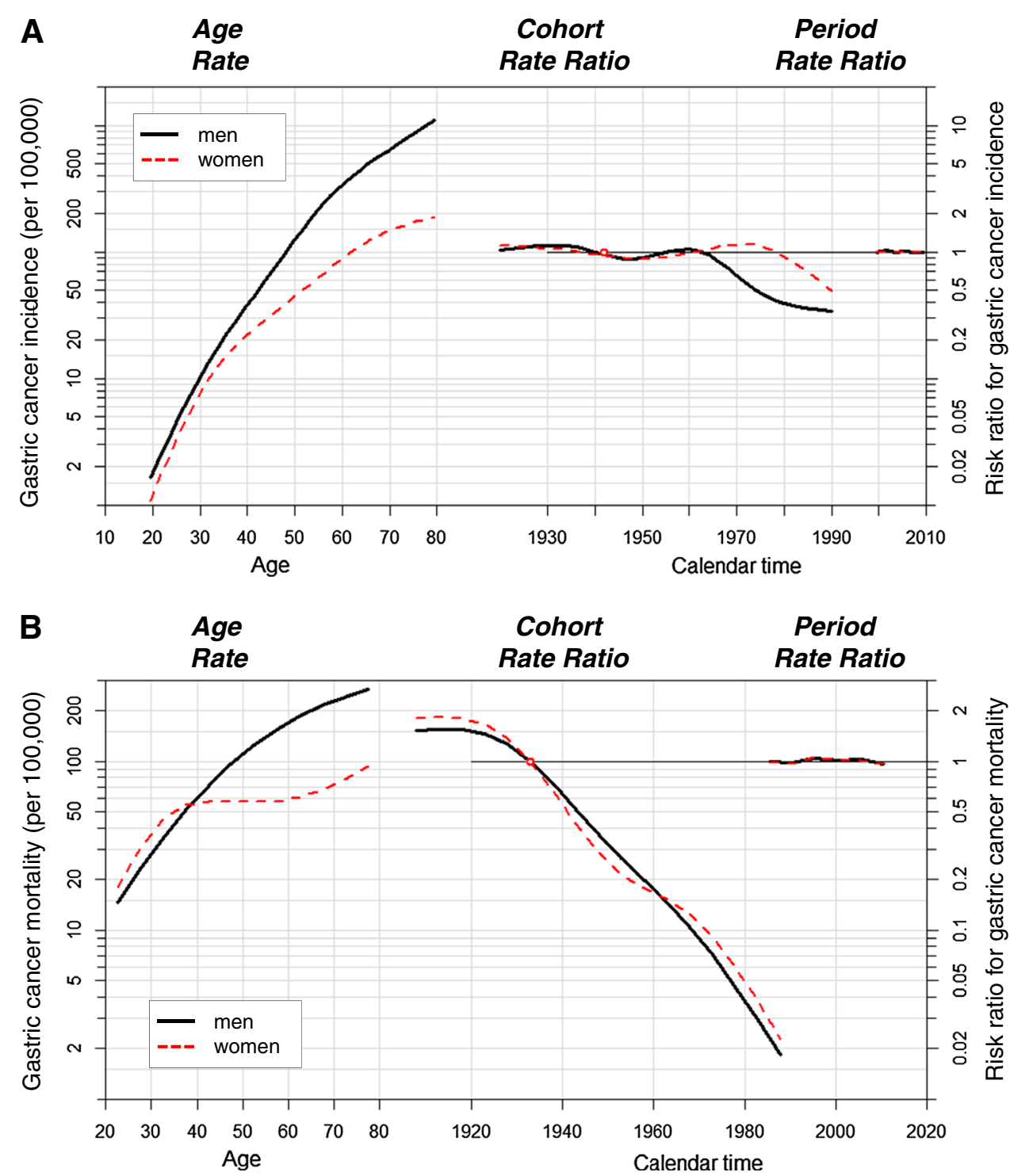

that the generations born after 1933 showed no downward trend of incidence in males and even an upward trend in females in the urban area Zaragoza [10]. In a study in the US, based on the National Cancer Institute's Surveillance Epidemiology and End Results (SEER) program, the incidence in those aged between 25 and 39 years in white males and females combined has increased significantly at $2.7 \%$ (95 \% CI 1.5-3.9); the result was restricted to the non-cardia gastric cancer incidence during the period 1997-2006 [9]. The APC analysis revealed an increasing incidence after the 1947 cohort, but no difference was observed between males and females in the pattern. As for the Asian population, Liu et al. reported a nonsignificant rise in the incidence of gastric cancer in the middle third of the stomach for females aged 30-49 in Japan during 1975-1989 [11].

As for the trend in mortality, Sonnenberg, using the data of four European countries (Denmark, England,
Switzerland and Italy), Japan and the US during periods as early as the 1920 s to 2007 , reported an increasing pattern in the cohorts born after approximately the 1930s in some populations after a steep fall took place around the late nineteenth century [8]. The standardized cohort-mortality ratios stratified by sex imply a differential pattern between males and females, but no statistics were presented in the article. The author then extended the analysis to six other European countries (France, Germany, The Netherlands, Scotland, Spain and Sweden), which revealed an increase in gastric cancer mortality in the latest cohorts [7]. An increase was not observed in any of the six countries, but most of them showed a halted or increasing trend in the generations born after the 1920s and 1930s. Data were not presented separately for each sex in the later report.

The present study found an increasing trend in incidence in the 40-59 age group. Contrary to the previous reports that found a rise or no downward trend in the youngest 
generations $[5,9,10]$, our results showed a decreasing trend in the 20-39 age group. The reason for the discrepancy can be considered according to several aspects. First, the study periods in the previous reports were earlier than in the present study, which was 1999-2010. When viewed according to the cohort effect, the generations experiencing an increase in incidence are those born during 1933-1954 in Spain, 1958-1981 in the USA and 1926-1959 in Japan. The subjects in the 20-39 age group in our study were those born between 1960 and 1990, so it can be speculated that later generations could have shown a different trend in other studies as well if they had been covered in the analysis. Second, the case number for the youngest generation may be too small to allow discussing the changes. The rates in the youngest generation were less than 10 per 100,000 person-years, which means even a smallest change in the number of cases could lead to a large variance in the AAPC. Thus, despite the fact that the AAPC rendered a statistically significant result, it should be safe to further determine the results with a longer duration. Third, contrary to the previous reports or limitations of the small number of cases in the 20-39 age group, the decrease in the youngest generation is perhaps a true phenomenon. Notwithstanding the few results mentioned above, there are still other subgroups showing a decreasing in trend even within the same studies. For instance, aside from the females from Zaragoza, the males from Zaragoza and males and females from Navarre were experiencing a decreasing trend in the youngest population in Spain [10].

Moreover, despite the downward trend in the youngest generation, the 40-59 age group in our study showed an increasing trend. This could be a similar phenomenon as in the other studies, especially in that the increase in trends was more pronounced in females. There have been numerous hypotheses on the dramatic decline in the incidence of gastric cancer, including a decrease in the prevalence of Helicobacter pylori (H. pylori) and increased use of refrigerators, which in turn had resulted in more consumption of fresh fruit and vegetables, etc. [2]. However, the causes of the enigmatic increase in the young populations observed recently are unknown. Studies speculate that recent changes in medical practice, including $\mathrm{H} 2$ blockers and proton pump inhibitors, have increased the pathogenicity of $H$. pylori, and there have been possible alterations in the risk factor patterns including obesity and changes in diet $[4,5,9]$. Korea is also experiencing a decline in $H$. pylori, whose prevalence decreased from $59.4 \%$ in 1995 , $53.6 \%$ in 2000 to $45.9 \%$ in 2005 [21]. Some risk factors, such as consumption of sodium [22], drinking alcohol [17] and obesity [23-25], have increased overall in Korea. Daily consumption of vegetables and fruits, which are considered probable protective factors, has been increasing [17]. In addition, the prevalence of other risk factors such as smoking has been decreasing [17]. However, the mixture of the effects of causative and protective factors cannot explain the macroscopic changes in incidence or mortality. Moreover, with limited data on the age-specific prevalence and change of these risk and protective factors, it is difficult to conclude how the specific agents have affected the age-specific discrepancy.

Another speculation that has not been discussed in depth in previous studies is a possible role of diagnosis or practice patterns. Korea has been providing a mass screening, recommending a biennial stomach cancer screening for those 40 years of age and older with direct upper gastrointestinal series (UGIS), endoscopy or both since 1999 [26, 27]. A recent publication by the Korean Gastric Cancer Association reports that the prevalence of surgically operated early gastric cancers (EGCs) has risen gradually from $28.6 \%$ in $1995,32.8 \%$ in $1999,47.4 \%$ in 2004 to $58.0 \%$ in 2007 [28]. Consequently, the higher prevalence of localized gastric cancer, which parallels the upward trend of EGC incidence, might also explain the decreasing mortality owing to the mass screening and the advances in diagnostic technology. Thus, the increased incidence in the 40-55 age groups and slowing rate of change in mortality may in large part be due to the increased number of EGCs found by mass screening programs in Korea. However, the period effect of screening could not be evaluated since the analysis period did not cover the period before screening had started.

The overall higher rate of gastric cancer incidence in males compared to females has inspired many researchers to search for variations in risk factors such as sociodemographic or lifestyle factors, as well as hormonal factors [29-32]. However, these studies have so far neglected the age-specific incidence to explain the differences, which is important, since the male predominance is mostly seen in the middle-aged and elderly groups [33]. Some have reported that the male predominance is a result of a 10-to 15-year delay in the onset of gastric cancer in females compared to males [34]. Gastric cancer in patients younger than 40 years old is thought to be associated with genetic changes rather than environmental factors compared to the older patients $[11,35]$. Thus, the recent increasing trend or, in our case, decrease in the youngest generation, which is distinct between males and females, suggests a differential effect of environmental change despite the underlying genetic effects. In Korea, a study from a nationwide survey of surgically operated patients reported that the proportion of middle third stomach cancers was the highest at $46.7 \%$ in patients aged less than 30 compared to $23.3 \%$ in those older than 70 , but no significant difference was found between males and females [28]. In the same study, compared to those aged 40 years and above, those younger than 40 years had a higher prevalence of undifferentiated 
histological type (86.1 vs. $53.9 \%, p<0.001)$ and diffuse type carcinomas (70.5 vs. $37.0 \%, p<0.0001)$, which are known to be less affected by environmental factors compared to intestinal types. However, whether those histological types were different between the sexes was not reported, nor were there any data to compare for the trend in the subtypes by sex.

This article illustrates the changing pattern in the agespecific trend in incidence and mortality of gastric cancer in males and females in Korea. The phenomenon of selected changes in the pattern of age-specific and sexspecific rates presents a challenge to epidemiological researchers as they try to address the causative roles and their interplay in depth. The study has the limitations of descriptive epidemiology using registry data, such as missing values, lack of individual-level information and nonstandardized pathological classification. Moreover, considerations of the geographical differences in etiology, diagnosis and treatment could not be covered because of limitations of data availability. Furthermore, we were not able to analyze the data according to different histological or anatomical subsites, which may have shown a differential pattern. Further studies evaluating age-period-cohort effects of incidence and mortality after data accumulation and analysis by histological and anatomical subsites by sex will be informative.

However, the strength of this study is the quality of the nationally representative data. The incidence data have a completeness of $97.1 \%$ determined by the Ajiki method by the year 2010, which guarantees a microscopic verification rate of $88.5 \%$; the death certificate only (DCO) rate is $1.4 \%[12,36]$. Furthermore, since Korea has a large enough data set, it was possible to conduct the entire analysis stratified by sex, which some of the previous studies investigating age-specific trends had failed to do. Since the epidemiology of gastric cancer incidence and mortality, as well as the risk factors, is thought to be different between males and females, it is important to assess the distinguishing pattern. Also, the analysis statistically confirmed the age group and sex interaction. Moreover, the change in incidence and mortality rate, as well as the differential pattern between the sexes, was backed up in the APC analysis.

In conclusion, in the midst of the overall decrease in the incidence and mortality of gastric cancer, a differential pattern among age groups was observed for each sex. Further studies are warranted to search for the specific pattern according to various histological or anatomical subsites, as well as the mechanism behind the differential pattern in the age and sex interaction in the trend of gastric cancer incidence and mortality.

Acknowledgments This research was supported by the BRL (Basic Research Laboratory) program through the National Research Foundation of Korea funded by the Ministry of Education, Science and Technology (2012-0000347) and by the National Research Foundation of Korea (NRF) A3 Foresight program.

Conflict of interest None declared.

\section{Appendix}

See Tables 2 and 3.

Table 2 Number of cases, age-adjusted incidence and mortality rates per 100,000 person-years and AAPCs of gastric cancer for 5-year age groups by sex in Korea during 1999-2010 (incidence) and 1983-2012 (mortality)

\begin{tabular}{|c|c|c|}
\hline & $\begin{array}{l}\text { Incidence AAPC \% (95\% } \\
\text { CI) }\end{array}$ & $\begin{array}{l}\text { Mortality AAPC \% (95\% } \\
\text { CI) }\end{array}$ \\
\hline \multicolumn{3}{|l|}{ Males } \\
\hline $20-24$ & $-6.5(-11.9$ to -0.8$)$ & $-8.9(-9.9$ to -7.9$)$ \\
\hline $25-29$ & $-6.1(-8.0$ to -4.2$)$ & $-7.3(-7.9$ to -6.6$)$ \\
\hline $30-34$ & $-3.3(-5.0$ to -1.5$)$ & $-6.3(-6.8$ to -5.8$)$ \\
\hline $35-39$ & $-3.1(-4.1$ to -2.1$)$ & $-6.1(-6.5$ to -5.7$)$ \\
\hline $40-44$ & $-0.1(-1.0$ to 0.8$)$ & $-6.7(-7.2$ to -6.1$)$ \\
\hline $45-49$ & $0(-0.5$ to 0.4$)$ & $-6.8(-8.0$ to -5.6$)$ \\
\hline $50-54$ & $0.4(-0.1$ to 0.9$)$ & $-5.8(-6.3$ to -5.2$)$ \\
\hline $55-59$ & $-0.5(-1.4$ to 0.4$)$ & $-5.8(-6.1$ to -5.5$)$ \\
\hline $60-64$ & $-0.5(-1.2$ to 0.2$)$ & $-5.5(-6.0$ to -5.1$)$ \\
\hline $65-69$ & 0.7 (0.1 to 1.2$)$ & $-4.6(-5.3$ to -3.8$)$ \\
\hline $70-74$ & $-0.2(-0.9$ to 0.5$)$ & $-3.3(-4.4$ to -2.2$)$ \\
\hline $75-79$ & $-0.9(-1.6$ to -0.2$)$ & $-1.5(-2.2$ to -0.8$)$ \\
\hline \multicolumn{3}{|l|}{ Females } \\
\hline $20-24$ & $-3.3(-7.1$ to 0.8$)$ & $-9.0(-10.1$ to -7.8$)$ \\
\hline $25-29$ & $-2.3(-3.9$ to -0.5$)$ & $-5.7(-6.4$ to -5.1$)$ \\
\hline $30-34$ & $-1.3(-2.6$ to 0.0$)$ & $-4.4(-4.9$ to -3.8$)$ \\
\hline $35-39$ & $0.3(-0.6$ to 1.3$)$ & $-4.1(-4.7$ to -3.5$)$ \\
\hline $40-44$ & $2.6(1.7$ to 3.5$)$ & $-4.9(-5.5$ to 4.3$)$ \\
\hline $45-49$ & $1.8(0.7$ to 2.9$)$ & $-5.4(-5.9$ to -4.9$)$ \\
\hline $50-54$ & $0.9(-0.1$ to 2.0$)$ & $-6.3(-7.0$ to -5.5$)$ \\
\hline $55-59$ & $-1.8(-2.7$ to -0.9$)$ & $-7(-7.4$ to -6.6$)$ \\
\hline $60-64$ & $-1.2(-2.1$ to -0.3$)$ & $-7(-8.1$ to -5.8$)$ \\
\hline $65-69$ & $-0.6(-1.3$ to 0.0$)$ & $-5.8(-6.3$ to -5.2$)$ \\
\hline $70-74$ & $-0.7(-1.5$ to 0.0$)$ & $-4.6(-6.5$ to -2.7$)$ \\
\hline $75-79$ & $-1.2(-2.2$ to -0.3$)$ & $-2.5(-4.1$ to -0.9$)$ \\
\hline
\end{tabular}

AAPC (average annual percentage changes) calculated using the Joinpoint Regression Program, version 4.0.4 
Table 3 Summary statistics for age-period-cohort models of gastric cancer mortality, by sex, Korea 1983-2012, aged 20-79

\begin{tabular}{|c|c|c|c|c|c|c|}
\hline & $d f$ & Deviance $(D)$ & $\triangle D$ & $p$ value for $\chi^{2}$ & Effect & Adjusted $R^{2}$ \\
\hline \multicolumn{7}{|l|}{ Incidence } \\
\hline \multicolumn{7}{|l|}{ Males } \\
\hline Age (A) & 180 & 1997.7 & & & & \\
\hline Age-drift (AD) & 179 & 1843.5 & 154.2 & $<0.0001$ & $\mathrm{D} \mid \mathrm{A}$ & 0.07 \\
\hline Age-period (AP) & 175 & 1793.2 & 204.5 & $<0.0001$ & $\mathrm{PIA}$ & 0.08 \\
\hline Age-cohort (AC) & 175 & 1109.1 & 888.6 & $<0.0001$ & $\mathrm{ClA}$ & 0.43 \\
\hline \multirow[t]{2}{*}{ Age-period-cohort (APC) } & 171 & 1063.8 & 45.3 & $<0.0001$ & $\mathrm{PIAC}$ & 0.44 \\
\hline & & & 729.4 & $<0.0001$ & ClAP & \\
\hline \multicolumn{7}{|l|}{ Females } \\
\hline Age (A) & 180 & 367.0 & & & & \\
\hline Age-drift (AD) & 179 & 356.3 & 10.5 & 0.0012 & $\mathrm{D} \mid \mathrm{A}$ & 0.02 \\
\hline Age-period (AP) & 175 & 343.6 & 23.4 & 0.0003 & $\mathrm{PIA}$ & 0.04 \\
\hline Age-cohort (AC) & 175 & 218.0 & 149.1 & $<0.0001$ & $\mathrm{ClA}$ & 0.39 \\
\hline \multirow[t]{2}{*}{ Age-period-cohort (APC) } & 171 & 204.9 & 13.1 & 0.1581 & $\mathrm{PIAC}$ & 0.41 \\
\hline & & & 138.7 & $<0.0001$ & ClAP & \\
\hline \multicolumn{7}{|l|}{ Mortality } \\
\hline \multicolumn{7}{|l|}{ Males } \\
\hline Age (A) & 66 & 39671.0 & & & & \\
\hline Age-drift (AD) & 65 & 5522.0 & 34149.0 & $<0.0001$ & $\mathrm{D} \mid \mathrm{A}$ & 0.86 \\
\hline Age-period (AP) & 61 & 4902.0 & 34769.0 & $<0.0001$ & $\mathrm{PIA}$ & 0.87 \\
\hline Age-cohort (AC) & 61 & 592.0 & 39079.0 & $<0.0001$ & $\mathrm{ClA}$ & 0.98 \\
\hline \multirow[t]{2}{*}{ Age-period-cohort (APC) } & 57 & 470.0 & 122.0 & $<0.0001$ & $\mathrm{PIAC}$ & 0.99 \\
\hline & & & 4432.0 & $<0.0001$ & ClAP & \\
\hline \multicolumn{7}{|l|}{ Females } \\
\hline Age (A) & 66 & 23592.5 & & & & \\
\hline Age-drift (AD) & 65 & 3625.4 & 19967.1 & $<0.0001$ & $\mathrm{D} \mid \mathrm{A}$ & 0.84 \\
\hline Age-period (AP) & 61 & 3271.5 & 20321.0 & $<0.0001$ & $\mathrm{PIA}$ & 0.85 \\
\hline Age-cohort (AC) & 61 & 401.0 & 23191.5 & $<0.0001$ & $\mathrm{ClA}$ & 0.98 \\
\hline \multirow[t]{2}{*}{ Age-period-cohort (APC) } & 57 & 315.0 & 86.0 & $<0.0001$ & $\mathrm{PIAC}$ & 0.98 \\
\hline & & & 2956.5 & $<0.0001$ & ClAP & \\
\hline
\end{tabular}

\section{Reference}

1. Ferlay J, S.I., Ervik M, Dikshit R, Eser S, Mathers C, Rebelo M, Parkin DM, Forman D, Bray, F. GLOBOCAN 2012 v1.0, Cancer Incidence and Mortality Worldwide: IARC CancerBase No. 11 [Internet]. Available from: http://globocan.iarc.fr. Cited 28 Dec 2013

2. Howson CP, Hiyama T, Wynder EL. The decline in gastric cancer: epidemiology of an unplanned triumph. Epidemiol Rev. 1986;8:1-27.

3. Kuller LH. Age-adjusted death rates: a hazard to epidemiology? Ann Epidemiol. 1999;9(2):91-2.

4. Correa P. Gastric cancer: two epidemics? Dig Dis Sci. 2011;56(5):1585-6 author reply 1586

5. Camargo MC, et al. Divergent trends for gastric cancer incidence by anatomical subsite in US adults. Gut. 2011;60(12):1644-9.

6. Botterweck AA, et al. Trends in incidence of adenocarcinoma of the oesophagus and gastric cardia in ten European countries. Int J Epidemiol. 2000;29(4):645-54.

7. Sonnenberg A. Time trends of mortality from gastric cancer in Europe. Dig Dis Sci. 2011;56(4):1112-8.

8. Sonnenberg A. Differences in the birth-cohort patterns of gastric cancer and peptic ulcer. Gut. 2010;59(6):736-43.
9. Anderson WF, et al. Age-specific trends in incidence of noncardia gastric cancer in US adults. JAMA. 2010;303(17):1723-8.

10. Aragones N, et al. Time trend and age-period-cohort effects on gastric cancer incidence in Zaragoza and Navarre. Spain. J Epidemiol Community Health. 1997;51(4):412-7.

11. Liu Y, Kaneko S, Sobue T. Trends in reported incidences of gastric cancer by tumour location, from 1975 to 1989 in Japan. Int J Epidemiol. 2004;33(4):808-15.

12. Jung KW, et al. Cancer statistics in Korea: incidence, mortality, survival and prevalence in 2010. Cancer Res Treat. 2013;45(1): $1-14$

13. Shin A, Kim J, Park S. Gastric cancer epidemiology in Korea. J Gastric Cancer. 2011;11(3):135-40.

14. Choi Y, et al. Long term trends and the future gastric cancer mortality in Korea: 1983-2013. Cancer Res Treat. 2006;38(1):7-12.

15. Lee HJ, Yang HK, Ahn YO. Gastric cancer in Korea. Gastric Cancer. 2002;5(3):177-82.

16. Shin HR, et al. Nationwide cancer incidence in Korea, 1999-2001; first result using the national cancer incidence database. Cancer Res Treat. 2005;37(6):325-31.

17. Korean Statistical Information Service (KOSIS). Available from: http://kosis.kr/statisticsList/statisticsList_01List.jsp?vwcd=MT_ ZTITLE\&parmTabId=M_01_01. Acessed 1 Jun 2013. 
18. Segi M. Cancer Mortality for Selected Sites in 24 Countries (1950-57). Japan: Department of Public Health, Tohoku University of Medicine; 1960.

19. Boyle P, Parkin DM. Cancer registration: principles and methods. Statistical methods for registries. IARC Sci Publ. 1991;95: 126-58.

20. Carstensen B, P.M., Hills M, Laara E, A package for statistical analysis in epidemiology. (R package version 1.1.49). 2013.

21. Kim JI, et al. Changing prevalence of upper gastrointestinal disease in 28893 Koreans from 1995 to 2005 . Eur J Gastroenterol Hepatol. 2009;21(7):787-93.

22. Anderson RN, Rosenberg HM. Age standardization of death rates: implementation of the year 2000 standard. Natl Vital Stat Rep. 3;47:1-16, 20.

23. Chow WH, et al. Body mass index and risk of adenocarcinomas of the esophagus and gastric cardia. J Natl Cancer Inst. 1998;90(2):150-5.

24. Khang YH, Park MJ. Trends in obesity among Korean children using four different criteria. Int J Pediatr Obes. 2011;6(3-4): 206-14.

25. Oh K, et al. Prevalence and trends in obesity among Korean children and adolescents in 1997 and 2005. Korean J Pediatr. 2008;51(9):950-5.

26. Leung WK, et al. Screening for gastric cancer in Asia: current evidence and practice. Lancet Oncol. 2008;9(3):279-87.

27. Nam SY, et al. Effect of repeated endoscopic screening on the incidence and treatment of gastric cancer in health screenees. Eur J Gastroenterol Hepatol. 2009;21(8):855-60.

28. Jeong O, Park YK. Clinicopathological features and surgical treatment of gastric cancer in South Korea: the results of 2009 nationwide survey on surgically treated gastric cancer patients. J Gastric Cancer. 2011;11(2):69-77.

29. Lindblad M, Rodriguez LA, Lagergren J. Body mass, tobacco and alcohol and risk of esophageal, gastric cardia, and gastric noncardia adenocarcinoma among men and women in a nested casecontrol study. Cancer Causes Control. 2005;16(3):285-94.

30. Freedman ND, et al. Male predominance of upper gastrointestinal adenocarcinoma cannot be explained by differences in tobacco smoking in men versus women. Eur J Cancer. 2010;46(13): 2473-8.

31. Camargo MC, et al. Sex hormones, hormonal interventions, and gastric cancer risk: a meta-analysis. Cancer Epidemiol Biomark Prev. 2012;21(1):20-38.

32. Chandanos E, Lagergren J. Oestrogen and the enigmatic male predominance of gastric cancer. Eur J Cancer. 2008;44(16):2397403.

33. Inoue M, Tsugane S. Epidemiology of gastric cancer in Japan. Postgrad Med J. 2005;81(957):419-24.

34. Sipponen $P$, Correa P. Delayed rise in incidence of gastric cancer in females results in unique sex ratio $(\mathrm{M} / \mathrm{F})$ pattern: etiologic hypothesis. Gastric Cancer. 2002;5(4):213-9.

35. Shiao $\mathrm{YH}$, et al. Microsatellite instability and/or loss of heterozygosity in young gastric cancer patients in Italy. Int $\mathrm{J}$ Cancer. 1999;82(1):59-62.

36. The Korea Central Cancer Registry, National Cancer Center. Annual report of cancer statistics in Korea in 2010. 